\title{
RESEARCH HIGHLIGHT pH-sensitive anti-CTLA4 antibodies: yes to efficacy, no to toxicity
}

\author{
Amnon Altman ${ }^{1}$ and Kok-Fai Kong ${ }^{1}$ \\ Cell Research (2019) 29:601-602; https://doi.org/10.1038/s41422-019-0198-8
}

\begin{abstract}
For a long time, anti-CTLA-4 antibodies were believed to mediate their beneficial cancer immunotherapeutic effect (CITE), whose effectiveness and safety are limited by associated immune-related adverse effects (irAE), via blocking the interaction between CTLA-4 and its ligands, a mechanism known as checkpoint blockade. Using novel engineered antibodies, Zhang et al. challenge this paradigm in the present study, demonstrating that the irAE of anti-CTLA-4 antibodies can be uncoupled from their CITE, raising hopes for the design of a new generation of safer and more effective anti-CTLA-4 antibodies.
\end{abstract}

Ever since its discovery in 1987, CTLA-4, a close relative of the prototypic $T$ cell costimulatory receptor CD28, has served as the prime example of a $\mathrm{T}$ cell inhibitory receptor. Its inhibitory role is evident from findings that CTLA-4-deficient mice display a severe immune dysregulation associated with a lethal lymphoproliferative disease, ${ }^{1,2}$ and that humans with haploinsufficient CTLA4 mutations display immune dysregulation that can be reversed by a CTLA-4-Ig fusion protein (Abatacept). ${ }^{3}$ However, the mechanisms underlying its immune inhibitory function are still being debated (reviewed in ref. ${ }^{4}$ ). Mechanisms that have been proposed include competition with CD28 for binding to their shared ligands, CD80 and CD86 (also known as B7-1 and B7-2, respectively), active delivery of inhibitory signals via the CTLA-4 cytoplasmic domain, and downregulation of costimulatory ligands on the surface of antigen-presenting cells (APCs). The complexity of CTLA-4 biology is exemplified by seemingly inconsistent findings that implicated either its extracellular domain or cytoplasmic tail as being critical for its inhibitory function (reviewed in ref. ${ }^{4}$ ). Further complicating the picture is the fact that in addition to T responder (Tresp) cells, in which CTLA-4 is induced by TCR stimulation, regulatory T cells (Tregs) constitutively express CTLA-4 at high levels. Moreover, CTLA-4 is required for the suppressive function of Tregs, ${ }^{5}$ and underlying this requirement, among others, is the ability of CTLA-4 to deplete CD80/86 from the surface of APCs, particularly dendritic cells (DCs) via transendocytosis, ${ }^{6}$ thereby preventing these APCs from efficiently activating tumor-specific Tresp cells.

CTLA-4 entered the tumor immunology scene when it was found that in vivo administration of anti-CTLA-4 antibodies enhances antitumor immunity. ${ }^{7}$ This finding prompted efforts to develop anti-CTLA-4 antibodies as immune-based therapies for cancer, and culminated with the approval by the FDA in 2011 of an anti-CTLA-4 antibody, Ipilimumab, for the treatment of unresectable advanced melanomas. Ipilimumab has displayed a substantial and durable cancer immunotherapeutic effect (CITE), and is now undergoing clinical trials in other cancers.
Nevertheless, the clinical use of Ipilimumab (and similar antibodies) in cancer immunotherapy is limited by the fact that it is effective in only a small fraction of cancer patients and, furthermore, it exerts a substantial and often severe immunotherapy-related adverse effect (irAE) in the form of inflammatory and autoimmune manifestations, necessitating cessation of treatment in some patients. Thus, there is an urgent need to understand the cellular and molecular basis of irAEs associated with the therapeutic use of anti-CTLA-4 antibodies. Such understanding would allow the development of antibodies that retain their much desired CITE, but are devoid of deleterious irAE.

For a long time, the therapeutic effect of anti-CTLA- 4 antibodies has been ascribed to their ability to block the interaction between CTLA-4 and its ligands, CD80/86, thus favoring the interaction of these ligands with the competing CD28 costimulatory receptor to result in effective activation of Tresp cells. This mode of action led to the concept of checkpoint blockade as the mechanistic basis for the CITE of anti-CTLA-4 antibodies, based on early findings that such antibodies blocked the binding of soluble CD80/86 to surface-bound CTLA-4. ${ }^{8}$ However, this model has recently been challenged by two 2018 reports, demonstrating that Ipilimumab did not, in fact, block the interaction of immobilized or cell-bound CD80/86 with CTLA-4, or the transendocytosis of these ligands from DCs. ${ }^{9,10}$ Furthermore, engineered antibodies that lost their ability to block the CTLA-4-CD80/86 interaction fully retained their CITE by depleting intratumoral Tregs. These studies further demonstrated that the desirable CITE can be uncoupled from the undesirable irAE. It is of interest that a novel Treg-intrinsic, CTLA-4-linked signaling pathway that we found to mediate contact-dependent suppression also appears to uncouple CITE from at least one form of experimental irAE, i.e., autoimmune colitis. $^{11}$

In an elegant study recently published in Cell Research, Zhang et al. took a substantial step forward by elucidating the molecular mechanism responsible for the irAE. ${ }^{12}$ They demonstrate that, while irAE-prone antibodies such as Ipilimumab directly associate with surface CTLA-4 for lysosomal degradation in a $\mathrm{pH}$ independent manner, the engineered non-irAE-prone antibodies dissociate from CTLA-4 after endocytosis in endosomal vesicles with lower pH (Fig. 1). The dissociated CTLA-4 subsequently interacts with LRBA, a vesicle trafficking chaperone, allowing its recycling to the cell surface. To further validate this mode of action, the authors went on to engineer Tremelimumab, an irAEprone anti-CTLA-4 antibody that has yet to gain FDA approval, to increase its $\mathrm{pH}$ sensitivity. This alteration allowed the resulting

${ }^{1}$ Division of Cell Biology, La Jolla Institute for Immunology, La Jolla, CA 92037, USA

Correspondence: Amnon Altman (amnon@lji.org)

Published online: 12 July 2019 


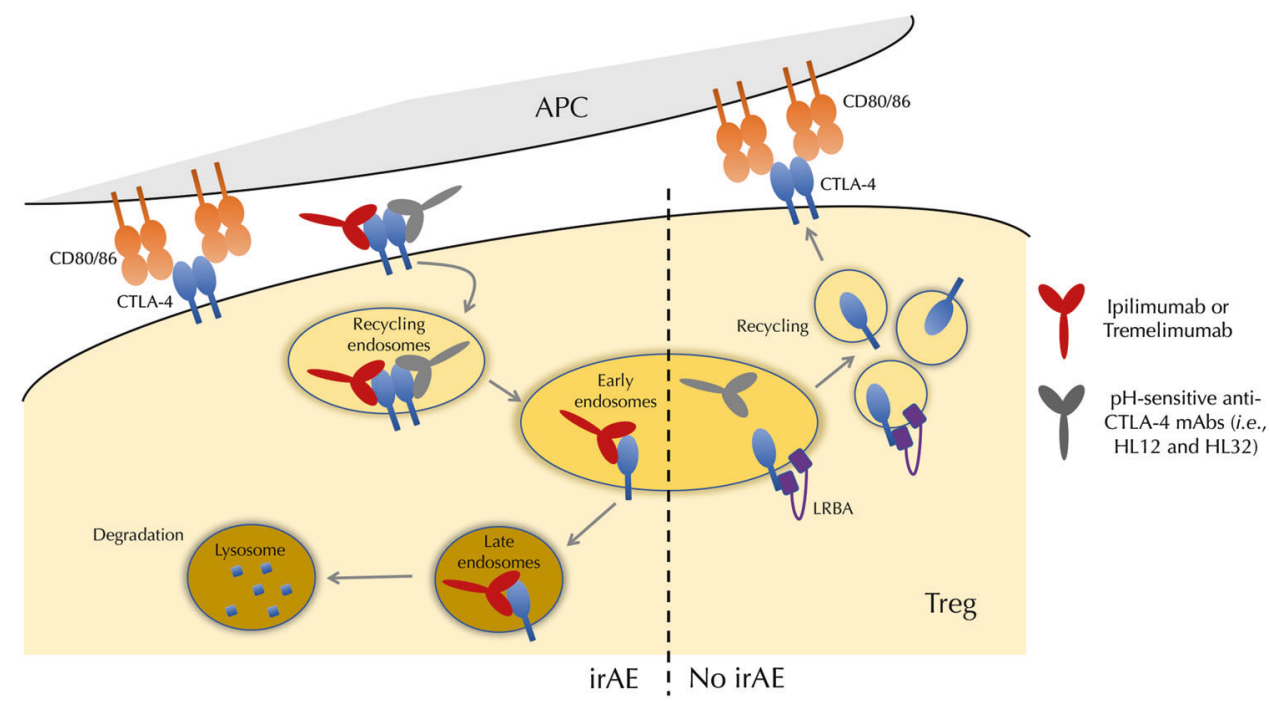

Fig. 1 Mechanisms of action of anti-CTLA-4 antibodies possessing or lacking irAE. When Ipilimumab and Tremelimumab, the prototypic irAEprone antibodies, bind to Treg surface-expressed CTLA-4, the complex is internalized into recycling endosomes that mature into early endosomes. These antibodies are insensitive to the early endosomal pH, remaining in a complex with the internalized CTLA-4. This prevents CTLA-4 from binding to the chaperone LRBA, a requirement for CTLA-4 surface recycling. Instead, the CTLA-4-antibody complex is targeted for degradation in the lysosome (left side of broken line in the figure). The loss of surface CTLA-4 promotes potent irAE, as exemplified in some cancer patients treated with Ipilimumab. Similarly, pH-sensitive antibodies such as HL12 and HL32 also bind to CTLA-4 expressed on the surface of Tregs and the CTLA-4-antibody complex is internalized into recycling endosomes, which mature into early endosomes having a pH of of 6.0-6.5. Unlike the pH-resistant Ipilimumab and Tremelimumab, however, at this $\mathrm{pH}$ HL12 and HL32 dissociate from the endocytosed CTLA-4, enabling CTLA-4 to bind to LRBA, which recycles CTLA-4 to the cell surface. This results in continuous CTLA-4 surface expression on Tregs (right side of broken line in the figure), which is required for protection against irAE. The continuous recycling and surface expression of CTLA-4 also allows for more stable antibody binding and Fc-dependent, ADCC-mediated depletion of Tregs, 9,10 leading to enhanced CITE

pH-sensitive antibodies to disengage from CTLA-4 in acidic endosomal vesicles, allowing CTLA-4 to recycle to the cell surface rather than being targeted for degradation. They also demonstrate that such antibodies display minimal, if any, irAE and, importantly, are more effective in Treg depletion and rejection of large established tumors in mice. Despite these encouraging in vivo studies, it remains to be determined whether similar $\mathrm{pH}-$ sensitive antibodies would exert a CITE in other tumors, especially in human cancer patients, since the acidic tumor microenvironment (a result of Warburg effect) may adversely affect the binding efficiency of such antibodies to CTLA-4. Nonetheless, these findings open the door for rationally designing tailor-made antibodies with abrogated irAE and enhanced CITE. The next obvious step would be to translate these exciting findings from basic discovery to clinical proof of concept. Hence, we can be cautiously optimistic about the future of anti-CTLA-4 cancer immunotherapy, which, despite perhaps not functioning via true "checkpoint blockade", could nevertheless represent an important step forward in immune-based cancer therapies.

\section{REFERENCES}

1. Tivol, E. A. et al. Immunity 3, 541-547 (1995).

2. Waterhouse, P. et al. Science 270, 985-988 (1995).

3. Lo, B. et al. Science 349, 436-440 (2015).

4. Walker, L. S. \& Sansom, D. M. Trends Immunol. 36, 63-70 (2015).

5. Wing, K. et al. Science 322, 271-275 (2008).

6. Qureshi, O. S. et al. Science 332, 600-603 (2011).

7. Leach, D. R., Krummel, M. F. \& Allison, J. P. Science 271, 1734-1736 (1996).

8. Keler, T. et al. J. Immunol. 171, 6251-6259 (2003).

9. Du, X. et al. Cell Res. 28, 416-432 (2018).

10. Du, X. et al. Cell Res. 28, 433-447 (2018).

11. Kong, K. F. et al. Nat. Immunol. 15, 465-472 (2014).

12. Zhang, Y. et al. Cell Res. https://doi.org/10.1038/s41422-019-0184-1 (2019). 